\title{
Complicações respiratórias pós-operatórias em cirurgia bariátrica: revisão da literatura
}

\author{
Postoperative respiratory complications in bariatric surgery: review of literature
}

Priscila Martins Delgado ${ }^{1}$, Adriana Claudia Lunardi ${ }^{2}$

Estudo desenvolvido na

Universidade de São Paulo (USP) São Paulo (SP), Brasil.

${ }^{1}$ Especialista em Fisioterapia Hospitalar pelo Instituto Central do Hospital das Clinicas da Faculdade de Medicina (FM) da USP - São Paulo (SP), Brasil.

${ }^{2}$ Fisioterapeuta do Departamento de Fisioterapia, Fonoaudiologia e Terapia Ocupacional da FMUSP São Paulo (SP), Brasil.

\section{ENDERECO PARA CORRESPONDÊNCIA}

Adriana Claudia Lunardi - Rua Cipotânea, 51 - Butantã - CEP: 05360-000 - São Paulo (SP), Brasil E-mail: adrianalunardi@usp.br

\section{APRESENTAÇÃO}

out. 2010

\section{ACEITO PARA PUBLICAÇÃO maio 2011}

FONTE DE FINANCIAMENTO nenhuma

CONFLITO DE INTERESSE nada a declarar
RESUMO: Obesidade é um problema de saúde pública mundial com altos índices de morbi-mortalidade. A cirurgia bariátrica tem sido o tratamento mais usado e desperta atenção para seu desenvolvimento e resultados. Porém, até o momento, não há revisão sobre a incidência de complicações respiratórias nesta população. $O$ objetivo deste estudo foi revisar a literatura sobre as complicações respiratórias após cirurgias bariátricas convencionais com a utilização das bases de dados PubMed, Cochrane e Scielo buscando os termos: complicações, pulmonar, pós-operatório e cirurgia bariátrica. Os limites foram os últimos dez anos, adultos, inglês e espanhol. Encontramos 69 artigos e utilizamos 21. As complicações respiratórias mais observadas em cirurgia bariátrica são: embolia pulmonar, atelectasias e pneumonia, estando relacionadas à idade e à hipoventilação. A obesidade mórbida está associada a disfunções respiratórias, incluindo diminuição da resistência cardiorrespiratória e dispneia, sendo as alterações mais comuns: diminuição da ventilação e da complacência torácica, taquipneia e aumento do trabalho muscular respiratório, com altos índices de hipoxemia e fadiga respiratória. Nossos resultados mostram que embolia pulmonar, atelectasias e pneumonias são as complicações respiratórias mais incidentes em cirurgias bariátricas convencionais, sendo os idosos ou portadores de síndrome da hipoventilação e apneia obstrutiva do sono os que apresentam maior risco de desenvolvê-las.

Descritores: obesidade; complicações; cirurgia bariátrica; pneumonia atelectasia pulmonar.

ABSTRACT: Obesity is a public health problem worldwide because of the high rates of morbimortality. Bariatric surgery has been the most often treatment and attracts attention on its development and results. However, there is no review about the incidence of respiratory complications in this population. The aim of this study was to review the literature on respiratory complications in conventional bariatric surgery utilizing the databases PubMed, Scielo and Cochrane. The terms searched were complications, pulmonary, postoperative care and bariatric surgery, and the limits, the last ten years, adults, English and Spanish. We found 69 articles, and used 21 , showing that the most common respiratory complications in bariatric surgery are pulmonary embolism, atelectasis and pneumonia, being related to age and the presence of hypoventilation. Morbid obesity is associated with respiratory dysfunction, including decreased cardiorespiratory endurance and dyspnea, being the most common changes: the decrease in ventilation and chest wall compliance, and tachypnea and respiratory muscle workload, with high rates of hypoxemia and respiratory fatigue. Our results suggest that pulmonary embolism, atelectasis and pneumonia are the pulmonary complications with the highest incidences in conventional bariatric surgery, and elderly and patients with hypoventilation or syndrome and obstructive sleep apnea have higher risk of developing postoperative pulmonary complications.

KeYwords: obesity; complications; bariatric surgery; pneumonia; pulmonary atelectasis. 


\section{INTRODUCÃO}

A obesidade é definida como um acúmulo de gordura generalizada ou localizada que causa danos à saúde. A morbi-mortalidade aumenta gradual e proporcionalmente ao índice de massa corpórea (IMC), reconhecido como padrão internacional de mensuração corporal e relativamente viável como indicador de sobrepeso e obesidade em adultos ${ }^{1}$.

Segundo estudos da Fundação Instituto Brasileiro de Geografia e Estatística (IBGE), há aumento constante da obesidade. Entre os homens, a prevalência foi de 5\% em 1989 e 9\% em 2002-2003, representando incremento de $4 \%$ ao ano. Já entre as mulheres, era 12\% em 1989 e 13\% em 2002-2003, ou seja, acréscimo de $0,4 \%$ ao ano $^{2}$.

A presença de alteração na mecânica respiratória desta população causa aceleração no padrão respiratório, tornando-o de baixa amplitude e provocando aumento no trabalho. A consequência disso é a redução de volumes e capacidades pulmonares, principalmente volume de reserva expiratória e capacidade residual funcional $^{3}$. Estas mudanças são ainda mais evidentes após o procedimento cirúrgico, especialmente quando realizado aberto e não por via laparoscópica ${ }^{4}$.

A cirurgia bariátrica como tratamento da obesidade mórbida deve ser considerada quando outras terapêuticas não foram efetivas e é indicada para pacientes com IMC maior que $40 \mathrm{~kg} / \mathrm{m}^{2}$ ou $35 \mathrm{~kg} / \mathrm{m}^{2}$ com comorbidade associada, como é o caso de problemas ostearticulares, hipertensão arterial sistêmica, diabetes mellitus, colecistopatia calculosa, apneia obstrutiva do sono ou dislipidemia ${ }^{5}$. Ela tem sido utilizada há décadas e um grande número de estudos tem mostrado sucesso terapêutico, porém, como toda cirurgia abdominal alta, não é isenta de complicações. As complicações respiratórias pós-operatórias estão entre as mais frequentes após cirurgias abdominais altas abertas e prolongam a estadia hospitalar, aumentam os custos e a morbi-mortalidade cirúrgica ${ }^{6}$.

Até o momento, desconhecemos qualquer publicação de revisões de literatura sobre a incidência de complicações respiratórias após cirurgias bariátricas convencionais e fatores relacionados. Nosso objetivo com o presente estudo foi revisar a literatura quanto às complicações respiratórias pós-operatórias mais comuns e aos fatores clínicos relacionados nas cirurgias bariátricas convencionais.

\section{METODOLOGIA}

A revisão foi realizada levando em conta as bases de dados PubMed, Cochrane e Scielo. A pesquisa incluiu a estratégia de texto livre por meio dos termos complicação pulmonar (pulmonary complications), pós-operatório (postoperative) e cirurgia bariátrica (bariatric surgery) nos últimos dez anos, seres humanos e adultos (idade entre 19 e 64 anos). Os idiomas considerados foram inglês e espanhol. A busca eletrônica foi realizada entre julho e setembro de 2008.

Para a seleção dos artigos, foram lidos os resumos, verificando se as informações preenchiam os critérios de inclusão: artigos completos de estudos transversais, prospectivos ou de revisão que citam algum tipo de complicação respiratória no pós-operatório (pneumonia, atelectasia, insuficiência respiratória e embolia pulmonar) de cirurgia bariátrica. Foram excluídos estudos de caso, estudos retrospectivos, textos contendo pacientes sem obesidade, cirurgia torácica, procedimentos cirúrgicos sem complicações respiratórias, cirurgia laparoscópica e artigos duplicados.

\section{RESULTADOS E DISCUSSÃO}

Foram encontrados 69 artigos, dentre os quais utilizamos 21. Os resultados foram agrupados nos tópicos: alterações na mecânica respiratória, valores espirométricos, complicações respiratórias pós-operatórias, apneia obstrutiva do sono e mortalidade.

\section{Alterações na mecânica respiratória}

São vários os fatores que interferem na mecânica respiratória do obeso, resultando em hipoventilação pulmonar. $\mathrm{O}$ excesso de tecido adiposo promove uma compressão mecânica sobre o diafragma, pulmões e caixa torácica, levando à restrição da mecânica pulmonar, provocando diminuição da complacência do sistema respiratório e aumento do trabalho respiratório, do consumo de oxigênio e do custo energético da respiração ${ }^{6}$. Essa restrição está diretamente relacionada ao IMC, sendo este um importante determinante dos mecanismos inspiratórios (trabalho feito por litro de ventilação) durante a anestesia geral com o paciente na posição supina ${ }^{7,8}$.

\section{Valores espirométricos}

A gordura armazenada na cavidade abdominal (ginóide) restringe a expansibilidade e reduz os volumes pulmonares ${ }^{7}$. Collins et al. ${ }^{8}$ observaram em um estudo transversal que o índice cintura/ quadril assume valores inversamente proporcionais aos volumes pulmonares ${ }^{8}$.

A disfunção diafragmática temporária, por longos períodos em decúbito dorsal horizontal, a dor e o uso de bloqueador neuromuscular também contribuem para redução da capacidade residual funcional, capacidade vital forçada e pressão parcial de oxigênio, devido à hipoventilação, causada pelo aumento da frequência respiratória, pela ausência de suspiros durante a anestesia e período pós-operatório imediato ${ }^{9}$.

Hamoui et al. ${ }^{10}$ observaram prospectivamente 146 pacientes submetidos à cirurgia bariátrica convencional e concluíram que a maior redução no volume corrente ocorre em pacientes com obesidade central, refletindo o aumento da pressão intra-abdominal e diminuição da complacência da parede torácica. A obesidade grave pode estar associada a alterações significativas na fisiologia cardiopulmonar, porém é múltipla e complexa. Até o momento, a cirurgia bariátrica tem se mostrado ser o tratamento mais eficaz, confiável e duradouro, já que proporciona perda de peso e, na maioria dos casos, resolve completamente os problemas de saúde pulmonar nos obesos mórbidos ${ }^{11}$.

\section{Complicações respiratórias pós-operatórias}

As cirurgias abdominais realizadas com incisão acima da cicatriz umbilical têm incidência de complicação pulmonar maior que as realizadas com incisões baixas, apesar da falta de padronização e consistência das definições das afecções consideradas complicações respiratórias. Isso ocorre pela perda da integridade da musculatura abdominal e consequente 
diminuição da força de contração, levando a alterações tóraco-abdominais por dor ou inibição reflexa ${ }^{12,13}$.

A principal e mais frequente alteração respiratória encontrada nesta revisão foi a espirométrica, o que deixa os pacientes mais susceptíveis a desenvolverem complicações pulmonares propriamente ditas. A principal redução relatada é a do volume corrente, relacionada a fatores como distúrbios do sono, altos valores de IMC e distribuição corporal do tipo ginóide. Já a complicação mais incidente foi a atelectasia, bastante comum e relacionada à presença de apneia obstrutiva do sono, ao tipo de anestesia utilizada e tempo de duração, como mostrado na Tabela 1.

Outra complicação respiratória comum após cirurgia bariátrica aberta é a pneumonia. Sua frequência é proporcional aos valores de IMC, assim como o risco de embolia pulmonar, que, além da relação com o IMC, também está associada à presença de distúrbio do sono, hipertensão arterial sistêmica e idade avançada. Em apenas um estudo desta revisão, a cirurgia bariátrica em pessoas com idade acima de 60 anos foi considerada tão segura quanto outro procedimento gastrointestinal, pois a mortalidade observada foi menor do que a esperada, embora a morbidade e a mortalidade sejam, em geral, maiores em idosos ${ }^{14}$.

\section{Atelectasias}

Não há dúvida que a incidência de complicação pulmonar pós-operatória está relacionada à presença de fatores de risco como tipo de anestesia utilizada, ato operatório e características inerentes ao paciente. A perturbação da mecânica respiratória produzida pela anestesia geral é mais pronunciada em pacientes obesos, já que esta induz atelectasia pulmonar $^{15}$, pois na população a anestesia acentua a redução da capacidade residual funcional, promovendo o fechamento precoce das pequenas vias aéreas ${ }^{16}$.

Ebeo et al. ${ }^{17}$ comparou a função pulmonar nos períodos pré e pós-operatório e observou redução de até 55\% da capacidade vital forçada e do volume expiratório forçado, sendo que até a anestesia epidural prejudicou a função respiratória de obesos ${ }^{18}$.

\section{Pneumonia}

Dentre as complicações respiratórias após cirurgia bariátrica aberta, a pneumonia é citada em $20 \%$ dos artigos revisados e sua incidência é de 2,4\%. Ela pode ser ocasionada pela diminuição do clearance mucociliar e efetividade da tosse, e aumento das secreções pulmonares ${ }^{19}$. Já nos superobesos, pacientes com IMC maior que $60 \mathrm{~kg} / \mathrm{m}^{2}$ submetidos à gastroplastia redutora, a incidência de pneumonia é de $3 \%$, sendo a complicação mais frequente a embolia pulmonar, 9\% dos $\operatorname{casos}^{20}$.

\section{Embolia pulmonar}

As comorbidades relacionadas à ocorrência de embolia pulmonar são: hipertensão arterial, Síndrome da Apneia Obstrutiva do Sono (SAOS), obesidade abdominal e alto valor de IMC. A embolia pulmonar é a complicação pós-operatória mais comum em pacientes obesos, como mostra a Tabela 121,22. Carmody et al. ${ }^{22}$ observaram 3.861 pacientes submetidos à cirurgia bariátrica e 0,9\% apresentaram embolia pulmonar, sendo estes com IMC significantemente maior em comparação aos pacientes sem tal complicação. Além

Tabela 1. Fatores relacionados à obesidade preditivos de complicações respiratórias pós-operatória

\begin{tabular}{|c|c|c|c|c|}
\hline & $\begin{array}{c}\text { Alteração } \\
\text { espirométrica }\end{array}$ & $\begin{array}{l}\text { Embolia } \\
\text { pulmonar }\end{array}$ & Atelectasia & Pneumonia \\
\hline Distúrbio do sono ${ }^{20,26,27}$ & + & + & + & - \\
\hline Anestesia geral ${ }^{14,15}$ & - & - & + & - \\
\hline $\mathrm{IMC}^{19,20}$ & + & + & - & + \\
\hline Distribuição corporal ${ }^{5,7}$ & + & - & - & - \\
\hline Idade $^{22}$ & - & + & - & - \\
\hline Hipertensão arterial20 & - & + & - & - \\
\hline
\end{tabular}

IMC: índice de massa corpórea disso, neste estudo, a embolia pulmonar causou óbito em $27 \%$ dos casos. Em outra pesquisa, Msika $^{23}$ relata mortalidade de 60 a $70 \%$ de $2 \%$ dos indivíduos acometidos por embolia pulmonar após cirurgia bariática $^{23}$, uma taxa muito elevada, se comparada à incidência de 0,3\% relatada num estudo de revisão americano publicado em $2005^{24}$.

Algumas características pré-operatórias, como mostra a Tabela 2, necessitam de maior atenção no período pré, peri e pós-operatório.

\section{Apneia obstrutiva do sono}

Estudos cruzados encontraram associação entre o aumento do peso corporal e a incidência de SAOS, sendo que a síndrome acomete $40 \%$ dos obesos ${ }^{25}$. Um estudo com 690 moradores selecionados aleatoriamente mostrou que o aumento de $10 \%$ do peso causou elevação do risco de desenvolvimento da apneia do sono em 6 vezes $^{25}$. Os pacientes com SAOS de longa duração podem desenvolver dessensibilização dos centros respiratórios para hipóxia e hipercapnia, resultando na síndrome de hipoventilação da obesidade, ou síndrome de Pickwick ${ }^{25}$.

A SAOS tem sido frequentemente associada a fatores cardiovasculares como hipertensão arterial sistêmica, metabolismo anormal de glicose e obesidade, com consequente aumento da morbimortalidade cardiovascular. Estes fatores de risco são componentes da síndrome metabólica ${ }^{26}$.

Martí-Valeri et al. ${ }^{27}$ avaliaram 184 pacientes submetidos à cirurgia de Capella, sendo que $23 \%$ apresentaram complicações respiratórias, dentre os quais $17 \%$ eram atelectasias diagnosticadas por meio de radiografia de tórax e $7 \%$ dessaturação arterial de oxigênio durante as primeiras horas após a cirurgia. Os autores também observaram que a perda de peso após a cirurgia melhorava os valores da gasometria arterial, da função respiratória e da polissonografia ${ }^{27}$.

Alteração da mecânica respiratória, apneia obstrutiva do sono e, menos frequentemente, hipertensão pulmonar e embolia pulmonar pós-operatória são as maiores contribuintes para pobres resultados pulmonares em pacientes obesos, sendo o posicionamento crítico 
Tabela 2. Incidência de complicações respiratórias no pós-operatório de cirurgia bariátrica convencional

\begin{tabular}{lrcccc}
\hline Autor & $\mathrm{n}$ & Seguimento & $\begin{array}{c}\text { Atelectasia } \\
(\%)\end{array}$ & $\begin{array}{c}\text { Pneumonia } \\
(\%)\end{array}$ & $\begin{array}{c}\text { Embolia } \\
\text { pulmonar } \\
(\%)\end{array}$ \\
\hline Nguyen NT et al. ${ }^{16}$ & 70 & 7 dias & 55 & - & - \\
Hamoui et al $^{10}$ & 146 & & - & - & - \\
Martí-Valeri C et al. $^{27}$ & 209 & 1 ano & 16 & - & - \\
${\text { Varela JE et al. }{ }^{14}}$ & 1339 & 30 dias & - & 4,3 & - \\
Nesset EM et al. ${ }^{36}$ & 1584 & 7 anos & - & 2,0 & 2 \\
DeMaria et al. ${ }^{15}$ & 2075 & 90 dias & - & - & 6 \\
Carmody BJ et al. ${ }^{22}$ & 3861 & 60 dias & - & - & 1 \\
Welle WE et al. ${ }^{19}$ & 7868 & & 2 & 2,4 & - \\
Byrne TK ${ }^{20}$ & & & - & 3,1 & 9,4 \\
Di Betta E et al. ${ }^{31}$ & 32550 & 7 anos & - & 4,7 & 9,4 \\
\hline
\end{tabular}

em pacientes com $\mathrm{SAOS}^{28}$. Porém, a maioria dos obesos mórbidos submetidos à cirurgia bariátrica apresenta resolução completa ou melhoria de comorbidades como diabetes, hiperlipidemia, hipertensão e apneia obstrutiva do sono, pela efetiva perda de peso $^{29}$.

\section{Mortalidade}

Assim como todo procedimento cirúrgico, o bariátrico também apresenta riscos e complicações, sendo a embolia pulmonar uma das principais causas de morte no pós-operatório desta cirurgia. Embora a sua incidência pareça estável, o número de cirurgias bariátricas realizadas anualmente está aumentando consideravelmente, tornando a embolia pulmonar fatal não rara. A incidência de mortalidade é maior em pacientes com idade entre 50 e 59 anos se comparados aos pacientes de 18 a 39 anos de idade ${ }^{23}$. As principais causas relacionadas à morte pós-operatória são: embolia pulmonar, fístula na anastomose e insuficiência respiratória, presentes em $80 \%$ dos óbitos nos 30 dias após o procedimento ${ }^{30}$.

Porém, fatores como escolha do procedimento, curva de aprendizado para a técnica, prevenção, diagnóstico precoce e tratamento de complicações cardiovasculares podem reduzir a mortalidade no pósoperatório $^{31}$. Segundo Jamal et al. ${ }^{32}$, uma mudança radical na filosofia do tratamento de obesos mórbidos deve ser oferecida antes da cirurgia bariátrica, e estratégias precisam ser desenvolvidas para diminuir o risco operatório, assegurando taxas atuais de $0,35 \%$ de mortalidade em centros de excelência, similar aos índices de complicações após colecistectomia ${ }^{33,34}$.

Evidências recentes mostram que a cirurgia bariátrica para obesidade grave está associada à diminuição da mortalidade global. Contudo, graves complicações podem ocorrer e, portanto, uma cuidadosa seleção dos pacientes é de extrema importância ${ }^{35,36}$.

\section{CONCLUSÃO E CONSIDERAÇÕES FINAIS}

São inúmeras as complicações respiratórias no período pós-operatório de cirurgias abdominais altas convencionais em qualquer indivíduo, sendo mais incidentes em obesos, devido às alterações respiratórias inerentes à obesidade e à presença de outros fatores de risco.

A obesidade associa-se a alterações da função pulmonar, sendo esta prejudicada à medida que o IMC cresce e, consequentemente, há aumento da incidência de complicação respiratória no pós-operatório, sendo as mais citadas na literatura: insuficiência respiratória, atelectasia, pneumonia, hipoventilação e embolia pulmonar.

Além do IMC, outras características são importantes de serem ressaltadas no pré-operatório, como idade, alteração da mecânica pulmonar, obesidade com distribuição corporal ginóide, SAOS, hipertensão arterial sistêmica, hipertensão pulmonar, diabetes e asma.

\section{REFERÊNCIAS}

1. House of Commons Health Select Committee. Obesity. Third Report of Session 2003/04. London: The Stationary Office; 2004.

2. Instituto Brasileiro de Geografia e Estatística (IBGE). Pesquisa de Orçamentos Familiares 20022003: análise da disponibilidade domiciliar de alimentos e do estado nutricional no Brasil. Rio de Janeiro: IBGE; 2004

3. Rigatto AM, Alves SCC, Gonçalves CB, Firmo JF, Provin LM. Performance ventilatória na obesidade. Saúde Rev. 2005;7(17):57-62.

4. Paisani DM, Chiavegato LD, Faresin SM. Volumes, capacidades pulmonares e força muscular respiratória no pós-operatório de gastroplastia. J bras pneumol. 2005;31(2):125-32.

5. Hanusch-Enserer U, Enserer C, Rosen HR, Prager R. Indication for bariatric surgery. Acta Med Austriaca. 2004;31(4):125-9.

6. Smetana GW, Lawrence VA, Cornell JE. Preoperative pulmonary risk stratification for noncardiothoracic surgery: systematic review for the American College of Physicians. Ann Intern Med. 2006;144(8):581-95.

7. Pelosi P, Croci M, Ravagnan I, Tredici S, Pedoto A, Lissoni $A$, et al. The effects of body mass on lung volumes, respiratory mechanics, and gas exchange during general anesthesia. Anesth Analg. 1998;87(3):654-60. 


\section{Referências (cont.)}

8. Collins LC, Hoberty PD, Walker JF, Fletcher EC, Peiris AN. The effect of body fat distribution on pulmonary function tests. Chest. 1995;107(5):1298-302.

9. Weller WE, Rosati C. Comparing outcomes of laparoscopic versus open bariatric surgery. Ann Surg. 2008;248(1):10-5.

10. Hamoui N, Anthone G, Crookes PF. The value of pulmonary function testing prior to bariatric surgery. Obes Surg. 2006;16(12):1570-3.

11. Davis G, Patel JA, Gagne DJ. Pulmonary considerations in obesity and the bariatric surgical patient. Med Clin North Am. 2007;91(3):433-42.

12. Baltasar A, Bou R, Bengochea M, Serra C, Pérez N. Mil operaciones bariátricas. Cir Esp. 2006;79:349-55.

13. Trus TL, Pope GD, Finlayson SR. National trends in utilization and outcomes of bariatric surgery. Surg Endosc. 2005;19(5):616-20.

14. Varela JE, Wilson SE, Nguyen NT. Outcomes of bariatric surgery in the elderly. Am Surg. 2006;72(10):865-9.

15. DeMaria EJ, Portenier D, Wolfe L. Obesity surgery mortality risk score: proposal for a clinically useful score to predict mortality risk in patients undergoing gastric bypass. Surg Obes Relat Dis. 2007;3(2):134-40.

16. Nguyen NT, Lee SL, Goldman C, Fleming N, Arango A, McFall R, et al. Comparison of pulmonary function and postoperative pain after laparoscopic versus open gastric bypass: a randomized trial. J Am Coll Surg. 2001;192(4):469-76.

17. Ebeo CT, Benotti PN, Byrd RP, Elmaghraby Z, Lui J. The effect of bi-level positive airway pressure on postoperative pulmonary function following gastric surgery for obesity. Respir Med. 2002;96(9):672-6.

18. Regli A, von Ungern-Sternberg BS, Reber A, Schneider MC. Impact of spinal anaesthesia on peri-operative lung volumes in obese and morbidly obese female patients. Anaesthesia. 2006;61(3):215-21.

19. Weller WE, Rosati C, Hannan EL. Predictors of in-hospital postoperative complications among adults undergoing bariatric procedures in New York state, 2003. Obes Surg. 2006;16(6):702-8.

20. Byrne TK. Complications of surgery for obesity. Surg Clin North Am. 2001;81(5):1181-93.

21. Brolin RE, Cody RP. Impact of technological advances on complications of revisional bariatric operations. J Am Coll Surg. 2008;206(3):1137-44.

22. Carmody BJ, Sugerman HJ, Kellum JM, Jamal MK, Johnson JM, Carbonell AM, et al. Pulmonary embolism complicating bariatric surgery: detailed analysis of a single institution's 24-year experience. J Am Coll Surg. 2006;203(6):831-7.

23. Msika S. Surgery for morbid obesity: 2. Complications. Results of a technologic evaluation by the ANAES. J Chir (Paris). 2003;140(1):4-21.

24. Poulose BK, Griffin MR, Zhu Y, Smalley W, Richards WO, Wright JK, et al. National analysis of adverse patient safety for events in bariatric surgery. Am Surg. 2005;71(5):406-13.

25. Vgontzas AN, Tan TL, Bixler EO, Martin LF, Shubert D, Kales A. Sleep apnea and sleep disruption in obese patients. Arch Intern Med. 1994;154(15):1705-11.

26. Shiina K, Tomiyama H, Takata Y, Usui Y, Asano K, Hirayama Y, et al. Concurrent presence of metabolic syndrome in obstructive sleep apnea syndrome exacerbates the cardiovascular risk: a sleep clinic cohort study. Hypertens Res.2006;29(6):433-41.

27. Martí-Valeri C, Sabaté A, Masdevall C, Dalmau A. Improvement of associated respiratory problems in morbidly obese patients after open Roux-en-Y gastric bypass. Obes Surg. 2007;17(8):1102-10.

28. Kaw R, Aboussouan L, Auckley D, Bae C, Gugliotti D, Grant $\mathrm{P}$, et al. Challenges in pulmonary risk assessment and perioperative management in bariatric surgery patients. Obes Surg. 2008;18(1):134-8.

29. Buchwald H, Avidor $Y$, Braunwald E, Jensen MD, Pories W, Fahrbach K, et al. Bariatric surgery: a systematic review and meta-analysis. JAMA. 2004;292(14):1724-37.

30. Virji A, Murr MM. Caring for patients after bariatric surgery. Am Fam Physician. 2006;73(8):1403-8.

31. Di Betta E, Mittempergher F, Nascimbeni R, Salerni B. Outcome of duodenal switch with a transitory vertical gastroplasty, in super-super-obese patients in an 8-year series. Obes Surg. 2008;18(2):182-6.

32. Jamal MK, DeMaria EJ, Johnson JM, Carmody BJ, Wolfe LG, Kellum JM, et al. Impact of major co-morbidities on mortality and complications after gastric bypass. Surg Obes Relat Dis. 2005;1(6):511-6.

33. Pories WJ. Bariatric surgery: risks and rewards. J Clin Endocrinol Metab. 2008;93(11 Suppl 1):S89-96.

34. Buchwald H, Estok R, Fahrbach K, Banel D, Sledge I. Trends in mortality in bariatric surgery: a systematic review and meta-analysis. Surgery. 2007;142(4):621-32.

35. Bult MJ, van Dalen T, Muller AF. Surgical treatment of obesity. Eur J Endocrinol. 2008;158(2):135-45.

36. Nesset EM, Kendrick ML, Houghton SG, Mai JL, Thompson GB, Que FG, et al. A two-decade spectrum of revisional bariatric surgery at a tertiary referral center. Surg Obes Relat Dis. 2007;3(1):25-30. 